\title{
4d Imaging of Polymer Electrolyte Membrane Fuel Cell Cathodes by Scanning X- Ray Microscopy
}

Juan $\mathrm{Wu}^{1}$, Adam P. Hitchcock ${ }^{1}$, Mirna Lerotic ${ }^{2}$, David Shapiro ${ }^{3}$, Viatcheslav Berejnov ${ }^{4}$, Darija Susac ${ }^{4}$ and Juergen Stumper ${ }^{4}$

1. Material Science \& Engineering \& BIMR, McMaster University, Hamilton, ON, Canada

2. 2nd Look Consulting, Hong Kong

3. Advanced Light Source, LBNL, Berkeley, CA, USA

4. Automotive Fuel Cell Cooperation Corp., 9000 Glenlyon Parkway, Burnaby, BC, Canada

Proton exchange membrane fuel cells (PEM-FC) are a promising green energy resource for automotive applications. The cathode is a key rate limiting component of PEM-FC. Typical cathodes are composed of carbon support particles, decorated with Pt catalyst, mixed with a perfluorosulfonic acid (PFSA) polymer. As the proton conduction media in the cathode, the distribution of the PFSA ionomer will affect PEM-FC efficiency, Pt utilization, and degradation kinetics. Optimization of ionomer loadings and distributions is a major goal of PEM-FC research. Scanning transmission X-ray microscopy (STXM) has been shown to be a very effective method to measure the ionomer in PEM-FC electrodes [1], with significantly reduced radiation damage and F-loss when compared to TEM-EELS or TEMEDX [2]. While most STXM studies of PEM-FC to date have used only 2D projection $[3,4]$, the real material is a complex structure where porosity and component distributions in 3D control device performance. Initial studies of PEM-FC materials by STXM tomography have been presented [5].

Measurements at a number of X-ray energies are the key to mapping ionomer in the cathode. If multiangle tomograms are measured at suitable X-ray energies, the results can be combined to determine 3D chemical maps, also called 4D imaging. To reduce the radiation damage to the highly radiation sensitive PFSA, a sparse set of rotation angles (as few as 10) and only 2 (or 4) energies are used. In order to extract valid results from these sparse data sets, a compressed sensing (CS) algorithm has been used, which has been shown to give much better reconstruction results rather than the more typical SIRT analysis with small numbers of tilt angles [6]. The STXM results show that, by reducing the incident flux and slight defocusing the spot size (to $50 \mathrm{~nm}$ from $30 \mathrm{~nm}$ ), the total radiation dose can be reduced to less than $20 \%$ of the critical dose for PFSA damage. Fig. 1a shows the F 1s spectrum of PFSA and the basis for 4D imaging ionomer, along with results from low-dose STXM tomography (Fig. 1b). Fig. 2 demonstrates low dose tomography - very little F 1s spectral change was observed after 3 successive 10angle, 2-E tomograms were measured on the same area.

We have also measured PEM-FC and related samples by ptychographic spectro-tomography. Ptychography is a scanned coherent diffraction imaging (CDI) technique which provides a spatial resolution exceeding the limitations of probe forming optics [7]. Soft X-ray ptychography has been developed recently at the ALS [7, 8]. The spatial resolution have been improved from the typical $30 \mathrm{~nm}$ of STXM to well below $10 \mathrm{~nm}$ - the published record spatial resolution is $5 \mathrm{~nm}$ [7]. Fig. 3 compares 2Dprojection maps of PFSA in a PEM-FC cathode, derived from STXM and ptychography. The superiority of the ptychography spatial resolution is evident, as is the similarity of the PFSA spatial distributions, once the spatial resolution difference is taken into account. Fig. 4 presents results of ptychographic spectro-tomography. Ptychography images were measured below and above the $\mathrm{F}$ 1s edge from $-66^{\circ}$ to $+60^{\circ}$ with $9^{\circ}$ increments. Carbon support maps were generated from the pre-edge absorption image; the 
ionomer map was generated from the F 1s difference signal [9].

\section{References:}

[1] D. Susac, V. Berejnov, and AP. Hitchcock, ECS Trans. 41 (2011), p. 629.

[2] L.GdeA Melo, et al, J. Power Sources 312 (2016), p. 23.

[3] D. Susac, et al, ECS Transactions 50 (2012), p. 405.

[4] A.P. Hitchcock, et al, J. Power Sources 266 (2014), p. 66.

[5] V. Berejnov et al, ECS Transactions, 50 (2012), p. 361,

[6] J. Wu et al, Microsc Microanal (2017) in review

[7] D.A. Shapiro et al, Nature Photonics 8 (2014), p. 765.

[8] S. Bea, et al, J. Am. Ceram. Soc. 98 (2015), p. 4090.

[9] Research supported by AFCC, NSERC CRD, CaRPE-FC and NSERC Discovery grants. The research was performed using the ambient-STXM at the Canadian Light Source, which is supported by CFI, and STXM5322, and the Nanosurveyor I on BL 5.3.2.1 at the Advanced Light Source, which is supported by DoE Basic Energy Sciences under contract No. DE-AC02-05CH11231.
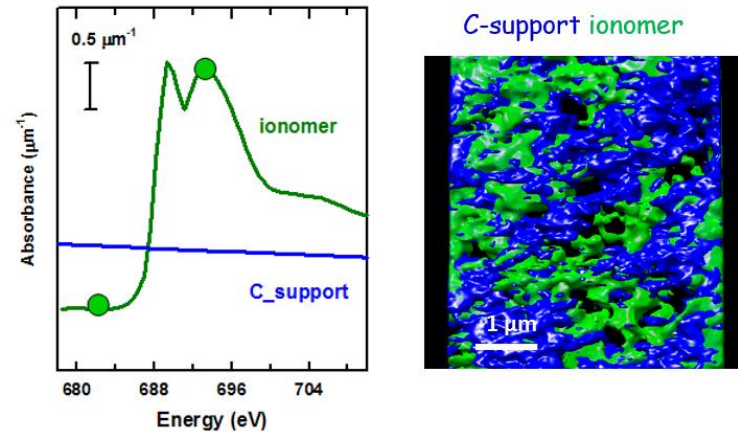

Figure 1. (a) F 1s spectra of PFSA, and carbon support.

(b) rendering from $3 \mathrm{D}$ reconstruction of PFSA distribution in a PEM-FC cathode. Differential imaging at 684 and 694 or $710 \mathrm{eV}$ is used to map ionomer (green) while C_support (blue) is deduced from the $684 \mathrm{eV}$ image.

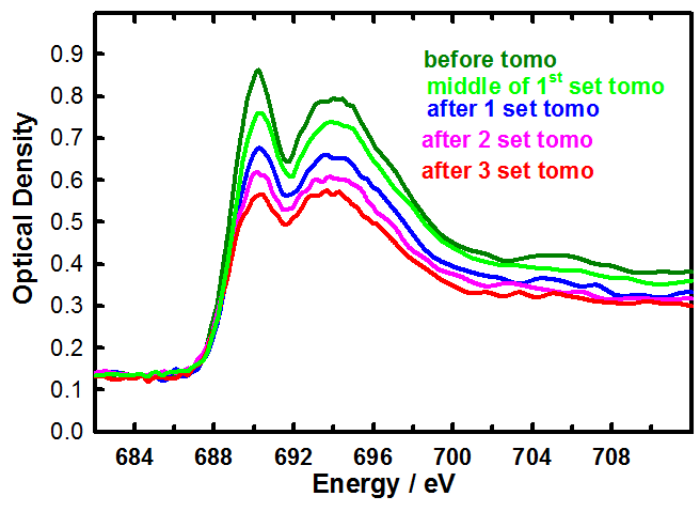

Figure 2. F 1s spectra of the tomogram volume determined before, during and after measuring each of three, 10-angle, 2-E tomograms.
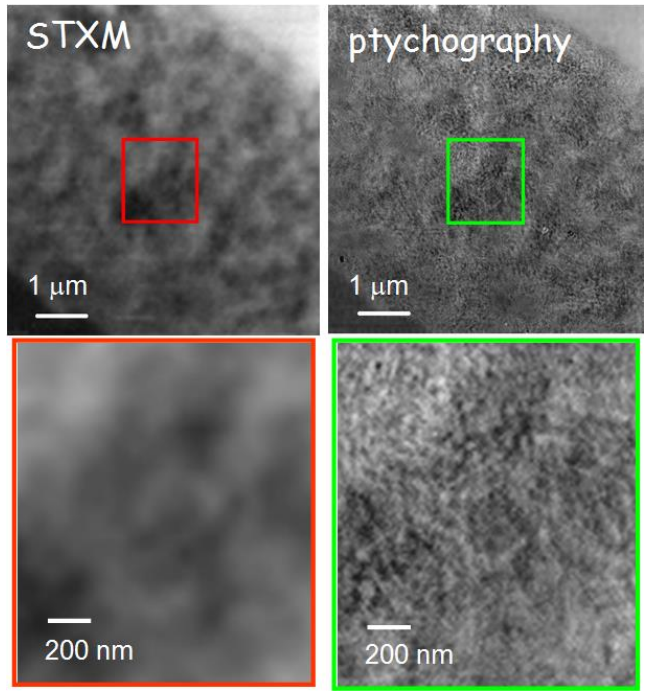

Figure 3. (left) STXM and (right) ptychography PFSA map of a portion of a PEM-FC cathode. The lower parts are an expansion of the areas outlined in red or green rectangles. The PFSA maps are derived from the difference in signals at $694 \mathrm{eV}$ and $684 \mathrm{eV}$.

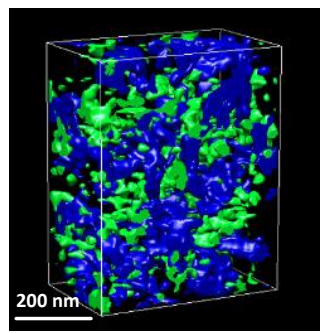

Figure 4. 4D ptycho-tomo imaging of ionomer (green) and carbon support (blue). 